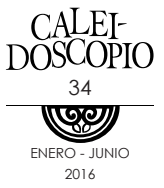

\title{
La contribución de tres aguascalentenses al entendimiento y la consolidación de la psicología en México,1849-1914
}

\section{RESUMEN}

A mediados del siglo xIx y principios del xx tres aguascalentenses, el licenciado Teodosio Lares, el doctor Jesús Díaz de León y el también licenciado Ezequiel A. Chávez, publicaron obras de divulgación de la psicología, en las que ofrecieron bien documentados recuentos de la concepción y los avances que se habían dado hasta su tiempo de y en esta ciencia; obras que tuvieron una gran importancia en la divulgación en México de los contenidos que habían de ser los cimientos sobre los que se logró su instauración. En este artículo, se ofrece un resumen de las aportaciones de estos tres pioneros de la psicología mexicana, y una comparación de cómo abordaron esta ciencia desde su saber y sus creencias.

Palabras clave: psicología, psiquiatría, alma, espíritu, conciencia.

1 Doctorante en ciencias sociales y humanidades por la Universidad Autónoma de Aguascalientes. Profesor de tiempo completo del Departamento de Formación Integral y Tutorías, Universidad Politécnica de Aguascalientes. eric.hidalgo@upa.edu.mx. Agradecimientos especiales al Consejo Nacional de Ciencia y Tecnología; a los doctores Andrés Reyes Rodríguez, Leticia Salazar Garza, Víctor Manuel González Esparza, Luciano Ramírez Hurtado y Miguel Ángel Sahagún Padilla, tutor de tesis y lectores; a los doctores José Antonio Saldívar Cervantes y Raúl Alejandro Gutiérrez García, por sus valiosas aportaciones. 


\section{ABSTRACT}

Between the end of the 19th century and the beginning of the 20th, three locals of Aguascalientes, Mr. Teodosio Lares, lawyer; Mr. Jesús Díaz de León, physician, and Mr. Ezequiel A. Chavez, also a lawyer, published written works that, seen through the eyes of the present, have had a great importance in the dissemination of the academic contents that form the core content of academic psychology, and that would ease the understanding and eventual implementation of this science in Mexico. The purpose of this article is to give a summary of the contributions of these pioneers of the Mexican psychology and a comparison of the way in which each of them conceived this discipline from the standing point of their knowledge and their religious beliefs.

Key words: psychology, psychiatry, soul, spirit, consciousness.

La UNAM, cuna de la institucionalización de la psicología en México, celebró en el año de 1996 los cien años del despegue de esta ciencia en el país con diversos eventos y la presentación de un libro: Cien años de la psicología en México (Álvarez y Treviño, 1997). En este libro, en el que se publicaron una serie de artículos, destaca uno que apunta esta efeméride. Fue en el año de 1896 que el licenciado en derecho Ezequiel A. Chávez, aguascalentense, presentara una propuesta para la modificación a los programas de la Escuela Nacional Preparatoria (ENP, la institución que sustituyó al Colegio Nacional de San Ildefonso y al sistema preparatorio eclesiástico que entonces funcionaba en el país) para que, entre las materias de ideología, materias pensadas para dar a los alumnos un bagaje filosófico, se impartiera la materia de psicología. Fue ésta la primera de grandes contribuciones que este personaje hizo a la instauración de la psicología en México (Menegus, 1984).

Chávez no fue ni con mucho el primer aguascalentense letrado en tener una comprensión avanzada a su tiempo de la psicología y en ayudar a su instauración en México. Cuarenta y siete años antes de las contribuciones de Chávez en este logro, se dio la publicación de un influyente libro para el propósito en México, el Tratado de psicología (sicología) del también licenciado en derecho Teodosio Lares (1849); y un año después de estas contribuciones (1897), se dio la publicación de un no tan influyente, pero sí bien realizado y documentado libro del localmente conocido doctor en medicina y hombre de ciencia, el doctor Jesús Díaz de León. En este artículo se ofrece una contextualización de las circunstancias 
en que fueron escritas estas obras y un análisis comparativo de sus contenidos.

\section{OBJETIVO}

El propósito de este artículo es ofrecer un análisis de tres obras de difusión de la ciencia de la psicología: Elementos de Sicología, del licenciado Teodosio Lares; El cerebro y sus funciones, del doctor Jesús Díaz de Léon; y An Outline of Psychology, del psicólogo Edward Titchener (traducida por el licenciado Ezequiel A. Chávez), producidas a lo largo del siglo XIX por tres individuos nativos del estado de Aguascalientes, México, que además fueron conocidos por su actuación en la política de su tiempo y en diferentes ámbitos; obras que ayudaron a la difusión de los contenidos básicos sobre los que se instauró la ciencia de la psicología en México. Además de los correspondientes análisis de las obras, el artículo intenta contestar las siguientes preguntas: ¿cómo concebía cada uno de estos autores la psicología?, ¿qué conocimientos tenían de acuerdo al avance de las ciencias auxiliares de la psicología hasta el momento de sus publicaciones?, y finalmente, ¿cómo influyeron estas obras en el entendimiento y la instauración de la psicología como ciencia en México entre mediados del siglo XIX y comienzos del XX?

\section{MÉTODO}

A lo largo del artículo se ofrecen análisis de las obras. Se respeta como orden de aparición el año de su publicación. Primero se ofrecen datos de los autores y del contexto en que se dieron las publicaciones; después, se ofrece un resumen de la forma en que están organizadas las obras y de los contenidos de sus diferentes capítulos. A través del desarrollo se ofrecen datos que ayudan a conectar los contenidos de las obras con investigaciones anteriores que dieron pauta a los autores para diseñar las concepciones bajo las cuales crearon los modelos explicativos que presentan, y comparaciones de la forma en que los tres autores concebían los contenidos que presentaron. Al final de la discusión de cada obra, se ofrece un pequeño análisis que ayuda a resumir las nociones vistas y a contextualizar al lector en lo que toca a datos comparativos de las mismas e información del impacto que tuvieron. 
Primera obra a ser analizada Elementos de psicología (sicología) del licenciado Teodosio Lares

El licenciado Teodosio Lares nació en el Mineral de Asientos de Ibarra, hoy día un municipio de Aguascalientes, en el año de 1806; estudió leyes y, debido a su habilidad para la argumentación legal, a sus amplios conocimientos en su ramo, en filosofía y en las diferentes ciencias que se iban conformando con el avance de la entonces naciente disciplina de la epistemología, tuvo un rápido ascenso en los sistemas legal y diplomático de México, pero siempre del lado de los gobiernos conservadores en tiempos en que México estaba luchando por lograr la definición de su forma de gobierno, razón por la cual ha sido relegado en la historia nacional que ha sido escrita bajo una visión liberal (Melgar, 2011).

El propósito con el que Lares escribió su hoy célebre obra de divulgación de la psicología fue el de proveer un texto para que los alumnos del Instituto de Ciencias de Zacatecas, a su cargo, tuvieran material para estudiar los contendidos que en el plan eclesiástico de estudios preparatorios de entonces se englobaban bajo la etiqueta de "ideología", y que habían sido pensados para prepararlos para el estudio de la filosofía. En la introducción a su tratado, Lares explica que, antes de que contaran con este material, estos alumnos se veían obligados a estudiar la ideología a partir de textos de Heineccio (1771), Altieri (1805) y otros autores, y que, por lo tanto, tenían que acudir a diferentes fuentes, lo que hacía el seguimiento de la materia complicado (Lares, 1849); además de este inconveniente, al acudir los contenidos del curso a la filosofía antigua, se hace obvio que su acervo promovía contenidos metafísicos de la ideología. Al crear un texto de "psicología", el autor impulsa el aprendizaje de contenidos documentados por autores cientificistas franceses, orquestados con apoyo en la naciente doctrina del positivismo, que estaba siendo propuesta, estudiada, explicitada y publicada por autores de la talla de Claude Henri Saint-Simon (1821), Auguste Comte (1853); más tarde de John Stuart Mill (1872), y más tratadistas que pasaron a la historia como los organizadores del conocimiento que abrió la puerta a la modernidad.

Para escribir esta obra, Lares (1849) explica abiertamente que basó sus contenidos en diferentes artículos y en un libro, publicados por diferentes autores franceses, mismos que menciona desde su largo título: Elementos de Sicología, formados de los diversos artículos sicológicos escritos en francés por Paffe, Jouffroy, Foscati, Satur, Benjamin Lafaye, Billot, Matter, Loyau 
D'Amboise, Saigey; y las obras filosóficas de Dougald Stewart. Traducidos al castellano, y ordenados en un cuerpo de doctrina. Para el uso de los alumnos del Instituto Literario de Zacatecas (Lares, 1849: 3-4). A pesar de ser un compendio de diferentes artículos y obras, el libro ofrece una unidad temática bien estructurada y una lógica tales, que se hace obvio que el autor escribió con soltura a partir de una noción bien fundada de los diferentes temas expuestos, idea que apoya Aceves (2001). La importancia de esta publicación estriba en que ha sido objeto de análisis por expertos en la historia de la psicología mexicana: (Aceves, 2001; De la Fuente et al., 1997; Gallegos, Colotla y Jurado, 1985; Pavón, 2011 ; Somolinos 1976).

\section{ANÁLISIS DE LOS CONTENIDOS DE ESTA OBRA}

Comienza el autor dando dos definiciones de psicología, la etimológica que al día de hoy sigue vigente y una más completa, que es la que le interesa dar a conocer: "Ciencia que trata del alma humana, de su origen, de los fenómenos que presenta en su estado actual y de su destino. Ciencia vasta por la multitud de hechos que abraza e importantísima porque debe resolver las cuestiones más interesantes para el hombre, las de su naturaleza y futuro destino" (Lares, 1849: 9).

En el tiempo en que la epistemología, la ciencia del conocimiento, estaba siendo establecida, Lares se preocupa por dar una justificación epistemológica para la psicología diciendo que "muchas cuestiones que quedaban dentro de los márgenes de su saber, relativas a las facultades intelectuales, se encontraban anteriormente en la lógica", pero que desde que progresos de la filosofía habían introducido divisiones más exactas, y aplicado denominaciones más convenientes a los diversos ramos de las ciencias, la "psicología" recobró su lugar como "fundamento de todas las teorías filosóficas" (Lares, 1849: 9-10), conclusión que, hoy es sabido, no está correctamente fundamentada.

De los autores en que Lares se apoyó para escribir su tratado, el más influyente es el francés C. M. Paffe (1832), quien acierta que la psicología se divide en dos ramos, el experimental y el racional, que este autor (1849) adopta.

El lado experimental de la "psicología", de acuerdo con esta visión, estudia los "hechos del alma humana", entiéndase por hechos todos los fenómenos que pasan por el espíritu humano o "teatro de la conciencia" (a lo largo de la obra se observa que Lares entiende por espíritu, por au- 
toconciencia y por alma la parte divina que habita en el ser humano) y que son observables, hechos como el que el ser humano ama, sufre, sabe (porque recuerda cosas), se mueve si quiere o se queda en un lugar. Los actos que resultan de todas las operaciones mentales del ser humano son observables y, por lo tanto, susceptibles de ser estudiados usando el mismo método de la observación, que es el método experimental; por lo tanto, su estudio se deja a la "psicología" experimental (Lares, 1849).

El lado racional de la "psicología" buscaría, por medio de la inducción y el raciocinio, dar cuenta de la división en el humano entre corporeidad y alma, y develar los misterios de ésta; parte notoriamente metafísica de una teoría supuestamente positivista de la psicología. El autor lo plantea de este modo: la psicología racional se ocupa "del origen, naturaleza y destino del alma humana" (Lares, 1849: 6-7), se ocuparía de explicitar esa dimensión.

A partir de aquí, el libro se divide en dos partes. En la primera el autor describe todos los contenidos correspondientes a la psicología experimental, apartado de 167 páginas; en la segunda se presenta el apartado correspondiente a la psicología racional, que consta de 72 páginas.

\section{Primer apartado: psicología experimental}

En lo que concierne a la psicología experimental, al hablar de los hechos del alma humana, los fenómenos que pasan por la conciencia, se hace claro que éstos son incontables. Todas las nociones, ideas, recuerdos, vivencias, problemas que llevan al humano a desarrollar una conducta observable se cuentan por miles, pero Lares explica que todos ellos pueden, inexorablemente y sin excepción, reducirse a tres órdenes: conocimientos, placeres (y penas) y actos, de los cuales derivan tres poderes o facultades del alma, la de conocer "conocimientos" (la inteligencia); la de gozar (o sufrir) "placeres y penas" (sensibilidad); y la de obrar "actos" (actividad). Intenta discernir nombres etimológicos para las ciencias que abarcarían estos contenidos, pero acaba llamando a estas disciplinas "teoría de la inteligencia", para conocimientos; "teoría de la sensibilidad", para placeres y penas; y "teoría de la actividad", para actos. El resto de esta parte primera lo dedica a la descripción detallada de los contenidos que sustentan la explicitación de cada una de estas teorías, mismos que se listan a continuación con una pequeña explicación (Lares, 1849). 
Sección I. Teoría de la inteligencia. La sección I del apartado de psicología experimental, teoría de la inteligencia, está dividida a su vez en nueve capítulos con esmeradas y completas explicitaciones de aquellos procesos mentales comunes a todo ser humano que denotan la existencia y el ejercicio de la inteligencia, sección de sesenta páginas (Lares, 1849).

Define la inteligencia como la facultad de conocer (recordar) y de elegir por medio del juicio o la comparación, el aquilatar. Siendo así, las facultades intelectuales son de dos clases: elementales (o perceptivas) "destinadas a darnos los conocimientos que nuestro entendimiento puede adquirir", y se dividen en la percepción externa, la conciencia, el juicio, la razón y el discurso; o reflexivas (adquirientes o modificadoras) "la habilidad de conservar conocimientos y de trabajar sobre ellos para combinarlos de diferentes maneras", y se dividen en la concepción, la asociación de ideas, la memoria, la imaginación, la abstracción, el lenguaje (Lares, 1849: 35).

Sección II. Teoría de la sensibilidad. Lares (1849) explicita entonces los hechos de la sensibilidad que, aclara, es como un complemento de la inteligencia y la actividad. La define como la susceptibilidad, de acuerdo a las circunstancias, de ser accesible al placer y al dolor. La sensibilidad hace del hombre una criatura completa, elevándolo de la vida vegetativa.

Esta sección consta sólo de dos capítulos, en los que el autor define también los conceptos de sensación: "modificación agradable o desagradable que nace en nosotros a consecuencia de un fenómeno orgánico", y de sentimiento: "todos los fenómenos afectivos, es decir todos los placeres y todas las penas que nacen inmediatamente de un fenómeno intelectual o de un fenómeno de actividad" (Lares, 1849: 102).

Sección III. Teoría de la actividad. Finalmente, pasa ocuparse de la actividad, esta sección consta de tres capítulos en los que el autor define tres formas de actividad: la actividad de la inteligencia, la que "dirige a la inteligencia hacia los objetos que le son estimables"; la actividad de los fenómenos sensibles, "a presencia de los objetos que son elementos de placer o sufrimiento, el alma no queda inerte, se dirige hacia ellos para aumentar su bienestar, y huye de los que le provocan sufrimiento"; finalmente define las afecciones o pasiones -que son causa de actividad-: "La tendencia del alma de dirigirse hacia los objetos de nuestros conocimientos para adquirir una idea más precisa y más completa; también de dirigirse hacia los objetos de nuestra simpatía para 
gozar; para poseerlos e identificarse con ellos" (Lares, 1849: 140-186). No se trata de actividad física; el alma es activa cuando se dirige hacia sus intereses, y pasiva si no lo hace.

\section{Segundo apartado: psicología racional}

Toca su turno ahora al lado racional de este sistema de estudio de la psicología, que buscaba teorizar acerca del "origen, la naturaleza y el destino del alma humana". Lares divide esta sección en tres capítulos: "del origen de nuestros conocimientos", "distinguir el espíritu de la materia" y "conocer su destino o estado futuro" (Lares, 1849); asuntos de difícil discusión, dada la naturaleza abstracta de la conciencia humana y el corte metafísico de estos temas.

Capítulo I. Del origen de nuestros conocimientos. El origen de nuestros conocimientos es el mismo que el de nuestras ideas y de nuestras facultades del entendimiento: "si el entendimiento tiene ideas, es deudor de éstas a las facultades en virtud de las cuales las tiene" (Lares, 1849: 189). ¿Cómo conocer los poderes de que el entendimiento está dotado? Sólo hay un medio: ir de los efectos a las causas, de los fenómenos a los principios. Los fenómenos son las ideas y los principios son las facultades mismas. Para discernir las facultades, se hace necesario estudiar las ideas mismas.

Capítulo II. Distinción del principio que piensa y la materia. Lares (1849) da por hecho que el ser humano está compuesto de un cuerpo y un alma, fuente de la voluntad y del pensamiento, principio de actividad, de sentimiento, de inteligencia y naturaleza antagonista de la materia. Hace una larga prosa acerca de cómo diferentes culturas y mitologías conciben al alma, su relación con el cuerpo y su destino al morir. En esta crítica deja patente su filiación a la religión católica (Lares, 1849).

Hace una crítica de teoristas materialistas como Hartley (1749/1834), Carlos Bonnet (1760), el doctor Gall (1825) y otros fisiólogos que en ese momento estaban buscando las correlaciones entre el funcionamiento del cerebro y el de diferentes órganos; acepta que han logrado un progreso, pero afirma que eso "no afecta al alma" (Lares, 1849). Lares hace mención de fisiólogos cuyas teorías manejaba, aunque en el momento en que estaba trabajando en su obra, las obras de los franceses Bichat (1832), Magendie (1884) y Bayle (1826) estaban causando una revolución en la fisiología y en la medicina. 
Capítulo III. Del destino futuro del alma humana. Lares (1849) afirma que, además del hecho de que el alma se manifiesta en el cuerpo, la certeza de su existencia después de la muerte tiene fundamentos como su razón y su libertad, más el derecho de la justicia divina sobre las acciones personales. Acierta que la inteligencia, la conciencia, las ideas e inclinaciones nada tienen de orgánico, lo cual sería una prueba de que el alma es inmaterial y puede existir sin el cuerpo. Si bajo ciertas enfermedades o la llegada a cierta edad la inteligencia y la coherencia de los hombres se ven disminuidas, esto se debe a que el alma, en diferentes partes de su conexión con el cuerpo, deja de tener un contacto y, por lo tanto, esas funciones se pierden para la materialidad (Lares, 1849).

\section{Importancia de los contenidos de la obra de Lares}

Como se puede observar, en este corto resumen de una obra de 260 páginas del licenciado Lares, los contenidos de la misma no dan cuenta de alguna forma de psicoterapia ni de contenidos de estudio del comportamiento en distintos escenarios, que es lo que se esperaría de una obra de psicología. En vez de esto, se pueden encontrar nombres y explicitaciones de diferentes procesos de pensamiento que lleva a cabo la mente en la vida común, cuestionamientos profundos acerca de la esencia de la conciencia, cualquiera que sea el nombre y la forma que se le atribuya, y datos filosóficos acerca de diferentes aspectos y concepciones de estas realidades. ¿Se puede decir que los contenidos de esta obra son de psicología? La respuesta a esta pregunta es sí. Meditando acerca de estos contenidos, se hace claro que la mayoría de las explicitaciones aquí compiladas nacieron de la filosofía, muchas de ellas de tradiciones metafísicas, pero fueron rescatadas por tratadistas con un ideario positivista de manera que se acercaran más a convenciones cientificistas. Cabe preguntarse, hoy que la psicología es una ciencia positiva, que se vale de la investigación en campo y de la discusión de casos clínicos para su avance, ¿̇los contenidos de este libro serían estudiados por ella? Sí, muchos de ellos forman parte del bagaje cultural del ciudadano común y muchos serían nociones básicas para cualquier profesional de la salud que se considerara abstracto y versado en la práctica de mejorar la vivencia de las personas a partir de ejercicios dialógicos (de la psicoterapia). En un tiempo en que la psicología era un campo del saber poco comprendido, el apren-

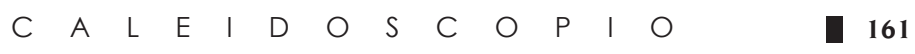


dizaje de las nociones compiladas por Lares se hacía importante a los futuros profesionales del país como nociones generales de cultura y como modelos de pensamiento-acción que les podían ayudar a tener una mente más penetrante y un buen sentido crítico.

Segunda obra a ser analizada: An Outline of Psychology del doctor Edward Bradford Titchener

El segundo de los tres aguascalentenses que contribuyeron de manera temprana a la difusión de la psicología en México fue el licenciado Ezequiel A. Chávez Lavista, hijo del ya entonces exgobernador de Aguascalientes Ignacio T. Chávez. El libro que aquí se analiza no es de su autoría y, antes de traducir éste, Chávez ya había participado en 6 publicaciones en el área de la psicología (Valverde, 1904), entre ellas los programas que, aprobados para la Escuela Nacional Preparatoria, significaron el comienzo de la enseñanza de esta ciencia en México.

Se trata aquí del libro An Outline of Psychology, escrito por el doctor en psicología Edward Bradford Titchener, quien fuera estudiante del filósofo y fisiólogo Wilhelm Wundt en la Universidad de Leipzig, Alemania. Wundt es considerado como el iniciador de la psicología científica positivista al haber creado la primera aproximación al estudio de la mente que se pudo considerar objetiva. Wundt propuso que era posible objetivar la vivencia de la conciencia bajo ciertas condiciones. Para el propósito, se hacía necesario poner a individuos entrenados a hacer diferentes tareas en diversas condiciones, a quienes se les pedía poner atención a las sensaciones y reflexiones que obtenían de éstas, para luego hacer recuentos detallados de las mismas. Años de este tipo de experimentación dieron a la ciencia las explicitaciones, las ideas y los métodos de trabajo que fueron el basamento bajo el que se iniciaron diferentes movimientos y corrientes psicológicas (Hothersal, 2005).

Titchener trajo todo este conocimiento a América cuando fue contratado como profesor por la Universidad de Cornell, Nueva York, donde desarrolló toda su trayectoria laboral (Hothersall, 2005). En Cornell, Titchener escribió el libro en cuestión como material para una de sus cátedras (Titchener, 1896), y Chávez se dio cuenta de que su estudio por parte de los estudiantes de nivel preparatorio en México como parte de las materias de "ideología" podía derivar en su desarrollo abstracto y de habilidades lógicas y personales, de manera que lo tradujo y, después de algunos años de impartir su curso de psicología, lo usó como material. 
Definición de psicología: desde el campo de la psicología científica, ésta puede ser definida como la ciencia de los procesos mentales. No debe ser definida como la ciencia de la mente o de la conciencia, porque estos fenómenos han sido estudiados (hasta entonces) por la metafísica (Titchener, 1896).

Definición de proceso en psicología: si la psicología es la ciencia de los procesos mentales, hay que definir a éstos. Un proceso (en psicología) es cualquier objeto de conocimiento científico que no es de naturaleza material. Un objeto material es permanente, cambia relativamente poco, pero se le puede ver como una unidad separada de los otros objetos a su alrededor. Un proceso es un movimiento hacia; es un progresivo convertirse en algo; una operación continua, que el observador científico puede seguir mientras se verifica. "La psicología estudia procesos y nunca estudia objetos materiales" (Titchener, 1896: 5).

La psicología trabaja con procesos: una precisión importante en este tema es el que una generalidad de personas piensa que la psicología trabaja con ideas. Las ideas tienen en la mente un cierto nivel de fijación. Si me agrada una persona, generalmente esa idea permanece igual, a menos que algo muy bueno o muy malo pase con ella. Los procesos no son fijos, hago un ejercicio mental para llegar a un resultado, opinión o forma de pensar, pero las ideas tampoco lo son, tienden a cambiar más lentamente; resultado de esto, se ve que "las ideas son también procesos" (Titchener, 1896: 6).

Cuando se pide a alguien llevar a cabo un proceso como dibujar algo, muchas ideas entran en juego: el papel que se va a usar, el instrumento de escritura, la forma en que éste se va a manejar, recuerdos del objeto a ser dibujado, etc. De esta manera, un proceso puede ser descompuesto en un número de ideas y procesos más simples (Titchener, 1896).

Justificación de la psicología como ciencia: de estas reflexiones ya se pueden obtener dos temas que son de interés para la psicología: 1. las influencias internas y externas que definen la especificidad última del dibujo; 2. estas influencias pueden ser separadas y clasificadas por el psicólogo $y$, dependiendo de su influencia en el resultado final, pueden recibir un peso. El sólo estudio bien estructurado de estas motivaciones da ya a la psicología el rango de ciencia, aunque la psicología experimental ha logrado mucho más (Titchener, 1896). 
Procesos mentales, conciencia y mente: la psicología es frecuentemente definida, por la gente en general y también técnicamente, como la "ciencia de la mente". La psicología puede aceptar esta definición si por mente se entiende la suma total de procesos mentales experimentados por los individuos a lo largo de su vida. El concepto de mente como es entendido por el común de la gente es que se trata de algo inmaterial o espiritual que se revela en nuestras ideas o sentimientos (idea que Lares defiende). Vista de esta manera, la mente sería un ente metafísico y, por lo tanto, no tiene cabida en la psicología (Titchener, 1896).

Esto da cabida a dos preguntas: ¿hay algo detrás de nuestros procesos mentales como una mente permanente? Y si la respuesta es sí, ¿cuál es su naturaleza? Para la psicología, aunque hallar la respuesta a estas preguntas pudiera parecer importante, ésta no es una de sus metas como ciencia, la psicología no ve en la mente más que la suma de los procesos mentales que se tienen a lo largo de la vida (Titchener, 1896).

El problema de la psicología: el objetivo del psicólogo es triple. Por una parte, busca descomponer la experiencia mental presente de los individuos en sus componentes más esenciales. Para lograr objetividad en esto, un proceso tiene que ser analizado más de una vez y por diferentes sujetos. Después, busca descubrir cómo estos elementos se combinan, cuáles son las leyes que gobiernan esas combinaciones. El proceso de la ira puede parecer simple, pero cuando se analiza el contexto en el que se da, la razón aducida, los acuerdos culturales implícitos que se rompieron para que una ofensa se verificara, es fácil darse cuenta de que descomponer la experiencia en sus elementos puede ser más complejo de lo que parece. Finalmente, la psicología busca hacer una conexión entre estos elementos y las condiciones fisiológicas que los llaman (Titchener, 1896).

En fin, el problema de la psicología es lograr describir y explicar procesos mentales. Esto implica capacidad de análisis y síntesis. Hace falta lograr una conexión entre los procesos mentales que llevan a diferentes reacciones, las circunstancias en que se dan y replicar esto hasta que se logren visiones objetivas a su respecto (Titchener, 1896). 
Capítulo II. La sensación como elemento consciente.

El método de investigar las sensaciones

Definición de sensación: cuando pensamos en algo, su recuerdo trae diferentes ideas como su olor, peso, color, el modo como lo conseguimos, nuestra opinión, entre otras. Si se medita acerca de tal objeto, pero la meditación se limita, por ejemplo, a aspectos visuales, se han aislado las sensaciones visuales que se tienen de él. Las ideas pueden traer consigo combinaciones de diferentes procesos mentales entre las que hay diferentes sensaciones de diferentes órganos de los sentidos. Una característica de las sensaciones es que, cuando han sido aisladas, se convierten en elementos simples que no pueden ser reducidos más adelante. De este modo, las sensaciones son "procesos conscientes elementales que están conectados con procesos corporales mediante órganos definidos del cuerpo" (Titchener, 1896: 26).

El método para investigar a las sensaciones: cada ciencia tiene sus objetos de estudio y su manera de aproximarse a ellos. El método utilizado por la psicología es el de introspección u autoobservación. Se trata de ver dentro de la propia mente o de las mentes de aquéllos a quienes se observa, para averiguar los procesos mentales que están activos a cada instante y cómo unos están influyendo a los otros. Este tipo de introspección no debe ser entendido como si la mente fuera una cosa con existencia separada y yo, el observador, pudiera tomar mi distancia y observarla desde afuera, lo que pasa es que el "yo", la observación y el fenómeno consciente bajo observación son todos procesos conscientes, de manera que cuando alguien se observa a sí mismo, un nuevo grupo de procesos se introducen a la conciencia del momento. Esta existencia de procesos no espontáneos cambia la naturaleza del proceso que deseo observar, hacerlo subjetivo y, por lo tanto, hacer este ejercicio uno fútil y sin importancia (Titchener, 1896).

De esta manera, la introspección no consiste en seguir un proceso durante su curso, sino en dejar que el proceso corra por entero permaneciendo atento al objeto del mismo que provoca la sensación. Este objeto es llamado estímulo (de cada sensación específica), y si se atiende a este estímulo, la sensación se hace más clara y tiene un lugar más duradero en la conciencia de lo que hubiera tenido si se hubiera dejado correr el proceso de manera espontánea (Titchener, 1896).

Lo que se ha descrito hasta esta parte son sólo los 4 primeros capítulos de una obra de 21 capítulos en total, pero una vez que se com- 
prenden los conceptos de idea, proceso e introspección en el contexto del libro, y la forma en que el método de la introspección buscaba llegar a la explicitación de la vivencia de la conciencia, el objetivo de este análisis se ha logrado, por lo que se pasa aquí al análisis de esta obra como aportadora de líneas de conocimiento que ayudaron a la comprensión e instauración de la psicología en México.

\section{Comentarios al libro de Titchener}

Como se puede constatar, Lares y Titchener (en las obras de su autoría estudiadas en este trabajo) tienen ideas diferentes acerca de la forma como debe proceder la psicología experimental de cara al avance de esta ciencia. Lares (1849) pensaba que la psicología experimental había generado por muchos años explicitaciones acerca de los diferentes fenómenos de la conciencia a partir de la observación del comportamiento y la documentación de categorizaciones filosóficas derivadas de estas observaciones. Titchener (1896), en apoyo al movimiento puro de la psicología experimental, avalaba el trabajo del laboratorio de Leipzig que había desarrollado métodos de trabajo y diseños a partir de la premisa de que, bajo ciertas condiciones y mediante cierto entrenamiento, se podía lograr que personas versadas en la explicitación de la conciencia dieran recuentos objetivos acerca de la vivencia de diferentes sensaciones después de experimentarlas en condiciones controladas. De esta manera, se buscaba explicitar, por ejemplo, a partir de cuántos grados de diferencia en temperatura los experimentadores podían discernir un cambio en esta variable; o las diferentes afectaciones fisiológicas, por ejemplo, de comer hielo; la forma en que la distancia de un objeto implica un cambio en el ángulo de la visión para mirarlo fijamente; miles más de experiencias que, siendo cotidianas, podían ser sujeto de explicitación. Después de algunos años de acumulación de este tipo de experimentos, el modelo de Wundt se agotó, pero los psicólogos que se formaron en su programa doctoral, diseñaron diferentes formas de experimentación que, al paso de los años han dado paso a todas las formas de investigación psicológica (Hothersall, 2005). 
Tercera obra a ser analizada: El cerebro y sus funciones; principios de psicología (y fisiología) basados en la anatomía de los centros nerviosos del doctor Jesús Díaz de León

El doctor Jesús Díaz de León fue un destacado ciudadano de Aguascalientes, miembro de su elite porfiriana. Estudió medicina en Guadalajara y de regreso a su estado ejerció su carrera, tuvo una trayectoria política y se dedicó a la promoción de la cultura mediante la edición de un periódico mensual de actualización científica, literaria, política y tecnológica, mediante el diseño de materiales didácticos y mediante la docencia (Gil, 2008).

El cerebro y sus funciones de Díaz de León (1897) demuestra el poder de observación de su autor y su conocimiento intuitivo o informado de modelos científicos que, años más tarde, iban a ser mejor conceptualizados y presentados al establishment científico por hombres de ciencia a los que se iba a dar crédito por su explicitación. Tratándose de un libro de divulgación y, debido a la necesidad del autor de poner diferentes temas psicológicos en contexto, el doctor Díaz de León se mete en la explicación de diferentes fenómenos físicos, químicos, psiquiátricos, sociales, científicos, etc. en los que se hace preguntas que iban a ser contestadas décadas más tarde. Cuestionamientos elegantemente puestos acerca de la difusión del calor y de la luz, de los mecanismos de la herencia, de la configuración del sistema nervioso, de las variables hereditarias, ambientales y sociales que moldean la personalidad, que sólo el hecho de que las haya formulado habla de su gran saber y su atinada sensibilidad para una gran diversidad de temas. Sigue aquí el análisis de su libro.

Capítulo I. El cerebro y sus funciones

Este capítulo es introductorio y da una idea de los contenidos de toda la obra. Explica el autor que desde las formas más simples de vida, plantas o animales que carecen de estructuras nerviosas y, por lo tanto, están sujetas a las condiciones externas de su medio para su supervivencia, a las formas de vida que las tienen, pero tan sólo con un desarrollo rudimentario y que les aseguran sólo un poder de reacción, hasta los animales superiores que cuentan con un cerebro sofisticado y con un sistema nervioso que consta de ramificaciones que conectan a este órgano con las diferentes partes del cuerpo, hay una gran diferencia en 
capacidad de reacción y poder de supervivencia y de adaptación al medio (Díaz de León, 1897).

El cerebro es, de todas estas estructuras que componen el sistema nervioso, la parte más misteriosa y la que más curiosidad científica ha despertado en la historia. Hace el autor un listado de los más destacados "cerebrófilos", la mayoría de ellos doctores franceses y alemanes que en ese momento estaban haciendo progresos importantes en la develación de los secretos de este órgano, del cual ofrece dos definiciones que denotan su admiración y curiosidad científica hacia éste: "El cerebro es el órgano que se considera como el lazo de unión entre la materia organizada, el cosmos que impresiona al sujeto y el espíritu" (definición que da idea de su visión del papel del cerebro como unión entre el espíritu y el cosmos). La segunda definición: "ese misterio grandioso al cual no ha podido llegar el escalpelo del anatómico, ni ha conseguido fotografiar la escuela espiritualista más minuciosa" (la escuela espiritualista afirma que el cerebro es la parte del ser humano que sirve como asiento del alma durante la vida terrena) (Díaz de León, 1897: 5-6).

El sistema nervioso, dice, es un poder central que, por medio de sus ramificaciones, conoce todos los actos del organismo. Por medio de los sentidos se pone en contacto con el exterior, pues éstos elaboran las sensaciones que viajan por estas ramificaciones y, de manera espiritualizada, son entregadas a la corteza cerebral, después de lo cual, lo que sucede con ellas está vetado al conocimiento del hombre, pero es sabido por la experiencia de la vida diaria que este órgano es responsable, además, de los procesos vitales de nutrición, de movimiento y de inteligencia que constituyen la vida (Díaz de León, 1897).

\section{Capítulo II. La materia, la fuerza}

En este corto capítulo de tan sólo cinco páginas, Díaz de León (1897) habla de diferentes fenómenos estudiados por la física y la química para ilustrar dos realidades de la psicología, la primera de estas realidades consiste en los estadios por los que tiene que atravesar la mente humana para obtener una correcta apreciación del tiempo y el espacio, aspectos del desarrollo de la inteligencia que más tarde estudiaría el psicólogo Jean Piaget (1929). Dice Díaz de León que el niño al nacer no tiene interés alguno en conocer estas dimensiones. Ya entrada la infancia carece aún de una idea del tiempo, por eso desea las cosas que 
pide al momento. Como va madurando, las regularidades de cada día le van enseñando el paso de las horas y el esfuerzo y el gasto de tiempo que debe hacer para obtener los objetos que le ayudan a adquirir una noción de espacio. Ver y tocar los objetos le da las ideas de peso, extensión y perspectiva que se van afinando con los años. Después hace comentarios de la manera en que esta forma limitada de percepción del niño se transforma en la sensibilidad del adulto.

\section{Capítulo III. Biogénesis}

Díaz de León (1897) se pregunta cuál ha sido el origen de la vida y si la inteligencia humana tiene la capacidad de descubrirlo. Da después una explicación a este respecto. La vida como hoy la conocemos pudo haberse generado a partir de una armonización de elementos químicos existentes en la tierra desde sus estadios primitivos. Criaturas más simples pudieron haber dado paso a criaturas más complejas como las cambiantes condiciones de la tierra lo fueron permitiendo (Díaz de Léon, 1897).

Explica que el discernimiento de los mecanismos de la vida era en ese momento causa de mucha curiosidad científica, pero que se había dado un gran paso con el descubrimiento de un compuesto llamado "microzyma", que es el material del que están hechos los tejidos de todos los seres vivos; explica, "Estos microzymas son en los vegetales y animales los trabajadores que en condiciones favorables se encargan de tejer las células" (Díaz de León, 1897: 17); esta teoría sostiene que los microzymas reunidos "secretan una cubierta y la célula queda formada"; de este modo cumplen su función, que es la de "construir los tejidos de los vegetales, de los animales y del hombre" (Díaz de León, 1897: 18).

Hoy que se han explicitado los mecanismos de la herencia, sabemos que no hay una sustancia que se organice sola para configurar los tejidos de todos los seres vivos. La realidad del microzyma de Béchamp es que este biólogo, experto en fermentación e investigador, hizo descubrimientos que parecían contradecir la teoría del también biólogo Luis Pasteur en cuanto a que las enfermedades son resultado del ataque de organismos exógenos. Béchamp (1883) propuso que el estrés de la vida diaria y la presencia de toxinas hacen que se generen ácidos al interior de los tejidos, lo cual es causa de la muerte de sus células. Cuando estas condiciones se dan, los microzymas se encargan de destruir las 
células desde el interior de los propios tejidos; mecanismo que ha sido desacreditado. Hoy se sabe que las infecciones sí tienen un origen exógeno (Hume, 1923).

\section{Capitulo IV. La vida celular}

En este pequeño capítulo de tres páginas, el autor ofrece una breve explicación de las estructuras que componen la célula; después fija su atención en las células que tienen a su cargo los procesos de sensibilidad y automatismo, las nerviosas, de las que pasa a ocuparse. Anota: "La vida de los individuos es la suma de las vidas individuales de los elementos que los componen" (Díaz de León, 1897: 24).

\section{Capitulo V. El sistema nervioso}

En este capítulo, el doctor Díaz de León (1897) vuelve a mostrar su asombro ante el cerebro y cita al influyente doctor y psiquiatra alemán Wilhelm Griesinger (1867), quien dice que, aunque podamos seguir la huella de una impresión, hasta que ésta se convierte en idea, una vez que lo hace, nunca podremos comprender lo que pasa con ella, pues el misterio de nuestro espíritu es incomprensible para nosotros. Al aliarse a esta idea, Díaz de León deja ver una vez más su creencia en la existencia de un alma como una entidad separada del cuerpo. López y De la Peña (2013) ha estudiado las creencias religiosas de Díaz de León, quien creía en la reencarnación.

En el siguiente párrafo, Díaz de León (1897) habla de otra aproximación al estudio de la mente desde el lado experimental (anteriormente se dio recuento de las de Lares y Titchener); habla de los resultados obtenidos desde el movimiento de la psiquiatría francesa por el doctor humanista Philippe Pinel (1800). Dice Díaz de León que el hecho de que los misterios encerrados en el diminuto órgano cerebral no permitan conocer la naturaleza real de la conciencia, "no quiere decir que los resultados de la observación de los fenómenos psíquicos sean estériles y casi imposibles de ser sujetados a leyes psicológicas"; "la patología ha llegado a descubrir muchas de éstas [leyes psicológicas] que la inducción y el método lógico habían sido impotentes para plantear" (Díaz de León, 1897: 26-27).

Lo que quieren decir estas afirmaciones es que la observación "objetiva" de los comportamientos de los individuos considerados locos 
(alienados), y los descubrimientos que se habían hecho desde esta observación, desde la fisiología y desde otros frentes, habían ya tributado resultados importantes en el estudio del comportamiento humano considerado anormal. Concreta estas afirmaciones diciendo: " $\mathrm{El}$ verdadero psicólogo debe ser un buen patólogo del sistema nervioso. La enfermedad es el mejor escalpelo [...] para conocer la constitución del sistema nervioso y sus funciones" (Díaz de León, 1897: 26-27).

Capítulo VI. Funciones de los elementos histológicos de los centros nerviosos

Como el nombre del capítulo lo dice, éste trata de las funciones de los diferentes tejidos del sistema nervioso humano. El autor explica que el elemento funcional de los centros nerviosos es la célula nerviosa (hoy conocida como neurona). La fibra nerviosa es sólo un medio de conducción del movimiento (sensorial/intelectual). Ejemplifica esto con las imágenes que recoge el ojo, traza la ruta que siguen hasta el cerebro donde pasan de ser movimiento fisiológico a ser movimiento psíquico (Díaz de León, 1897).

Habla el doctor Díaz de León (1897) de diferentes realidades fisiológicas que contribuyen al mantenimiento de la vida y deriva de todos estos modelos nociones de la conciencia que dan a entender cómo la biología del cerebro es la base para la generación de la personalidad y de la viabilidad intelectual de los seres humanos. Dice este hombre de ciencia: "Entre las operaciones más delicadas que tiene que llevar a cabo la actividad nerviosa de las regiones corticales del cerebro está la génesis de la noción de la personalidad, la conciencia del yo, la personalidad humana, que son un corolario de la sensibilidad propia de la célula nerviosa" (Díaz de León, 1897: 61).

Ofrece después esquemas de pensamiento que buscan discernir la diferencia entre normalidad y locura de la mente humana; por el lado de la normalidad dice que el humano está dotado de autoconsciencia, o sea, la capacidad de coordinar sus recuerdos y conocimientos para saber que se trata de sí mismo y actuar bajo este tenor; del otro lado está la "locura": la substancia gris superficial o cortical es el centro de la inteligencia, porque es generadora de las ideas. El hombre utiliza las impresiones recibidas, las cuales coordina; de su afortunada coordinación es de donde resulta la noción de personalidad. Un demente, un loco, un enfermo del cerebro no es capaz de lograr una afortunada coordinación de impresiones pasadas y presentes y, por 
lo tanto, no puede ser considerado como una persona jurídicamente (Díaz de León, 1897).

\section{Capítulo VII. La palabra}

En este capítulo el doctor Díaz de León (1897) hace una cuidadosa y afortunada descripción del fenómeno del habla humana en la que ofrece nociones conocidas que no aportan nada al objetivo de este resumen (Díaz de León, 1897).

Capítulo VIII. Los agentes físicos impresionales y la transmisión del movimiento expresivo

Igual que el capítulo anterior, éste es uno en que el doctor Díaz de León (1896) ofrece nociones que, para el tiempo en que el libro fue escrito, eran avanzadas, y hoy su descripción sería trivial. Habla de los fenómenos físicos que involucran el contacto de las personas con el medio ambiente por medio de los sentidos.

\section{Capítulo IX. La trasformación del movimiento}

En este último capítulo el doctor habla de la teoría de Laviosier de la conservación de la materia y hace reflexiones filosóficas que tienen que ver con ésta y el devenir de la vida (Díaz de León, 1896).

\section{Comentarios a la obra del doctor Jesús Díaz de León}

Jesús Díaz de León, quien dedicaba una gran parte de su tiempo, dinero y atención a permanecer actualizado en las cuestiones del saber en todas las áreas como se iban generando avances en el mundo, cuando se propone escribir una obra de psicología piensa en los contenidos psicológicos que puede derivar de los modelos sociales, científicos, tecnológicos y, sobre todo, médicos, que ya conoce, y de esta manera, produce una obra en la que habla de psicología desde la multidisciplinariedad. Así, habla de psicología cuando ofrece definiciones de las dimensiones de espacio y tiempo, y explica cómo la percepción del humano en estas dimensiones es casi nula en la infancia y se va refinando como las personas crecen en edad y madurez; habla de psicología cuando ofrece un estudio comparativo de los sistemas nerviosos 
de diferentes animales y explica cómo la mayor sofisticación de estas estructuras mejora la habilidad de supervivencia y adaptación de diferentes especies; habla de psicología (más por el lado de la psiquiatría) cuando explica la conformación del sistema nervioso y la manera en que diferentes enfermedades, lesiones y estilos de vida le afectan, perjudicando a la vez la conducta de maneras que pueden ser estudiadas; habla de psicología cuando busca explicar la manera concertada en que funcionan los diferentes componentes del sistema nervioso y cómo éste recoge impresiones del medio, las "espiritualiza" y las envía al cerebro, en donde, según él, ya no se pueden monitorear. Esta labor multidisciplinaria es, sin duda, una gran contribución al entendimiento e instauración de la psicología en México, aunque hoy no se sepa mucho acerca de la difusión que pudo llegar a tener.

\section{CONCLUSIONES}

Entre mediados del siglo XIX y principios del XX, hubo tres aguascalentenses cultos. Los tres fueron profesionistas en tiempos en que pocos alcanzaban esa distinción; dos licenciados en derecho, uno doctor en medicina. Los tres políticos y con experiencia en el Congreso Mexicano. Uno de ellos de cuna humilde, Teodosio Lares, los otros dos hijos de familias de elite en el estado, los tres actualizados hasta su tiempo en la producción de las obras filosóficas y científicas que se iban produciendo en Europa; con una amplia cosmovisión, tenían ideas sociales de avanzada e ideas de piedad tan grandes que tomaron como sus objetivos de vida el mejoramiento de su nación desde diferentes trincheras. Estos tres individuos escribieron obras para promover el conocimiento de los múltiples y abstractos escenarios en que la psicología toma forma (aunque en este trabajo la obra analizada por parte del licenciado Ezequiel A. Chávez fue escrita por otro personaje, después de su experiencia docente en la ENP, Chávez escribió obras psicológicas de gran trascendencia en México y otras partes del mundo); obras que contribuyeron a la difusión y el entendimiento de estos saberes, lo cual contribuyó, a su vez, al avance del conocimiento científico y filosófico en México. Las obras de los licenciados Teodosio Lares (1849) y Edward Titchener habían sido ya objeto de análisis en obras anteriores, la del doctor Díaz de León necesita y merece mucho análisis, dadas la complejidad y bastedad de sus conocimientos y la intención con la que 
fue escrita. El hecho de que estos tres aportadores al entendimiento y la consolidación de la psicología en México hayan nacido en territorios que hoy conforman el Estado de Aguascalientes, puede ser visto como una coincidencia, pero el análisis comparativo de sus obras por sí mismo y dada esta realidad da materia para la justificación de un artículo y probablemente obras de análisis más extensas.

\section{REFERENCIAS}

Aceves, E. (2001). Teodosio Lares, elementos de psicología. En M. Rovira, Pensamiento filosófico mexicano del siglo XIX y primeros años del XX. Vol. 3, pp. 73-93, México: UNAM.

Altieri, L. (1805). Elementa philosophiae ex probatis autctoribus adornata. Venecia, Italia: Tipografía de Antoni Zatta.

Álvarez, G. y Treviño A. (1997). En busca de la identidad: hacia la fundación de psicología de la UNAm 1959-1973. En J. J. Sánchez. 100 años de la psicología en México (69-84). Facultad de Psicología, unAm: México.

Bayle, A. (1826). Traité des maladies du cerveau et de ses membranes; maladies mentales. Philadelphia: Grigg.

Béchamp, A. (1883). Les microzymas dans leures rapports avec l'hétérogénie la physiologe et la pathologie. Paris: Librarie J.-B. et fils.

Billot, F. (1831). Barreau et de la magistrature suivis dún essai sur les jurisdictions. Paris: Chez M. Aubin, libraire, sur le cours.

Bichat, X. (1832). Oeuvres complétes de Bichat. Paris: J.-S. Chaudé, Librarie-Éditeur.

Bonnet, C. (1760). Essai analytique sur les facultés de l'âme. Copenhagen: Chez le freres C. \& A. Philibert.

Comte, A. (1853). The positive philosophy. Londres: John Chapman.

D'Amboise, L. (1838). Le Voeu Des families on une digue aux mauvais romans. Paris: Chez Angé editeur, rue Guénégaud.

De la Fuente Muñiz, R., Medina-Mora, M. E., y Caraveo, J. (1997). La salud mental en México. México: FCE.

Díaz de León, Jesús. (1897). El cerebro y sus funciones; principios de psicología basados en la anatomía de los centros nerviosos. Aguascalientes: Est. Tip. De Ricardo Rodríguez Romo.

Foscati, D. (1827). De la necessité d'etudier une nouvelle doctrine, avant de la juger, et application de ce principe à la physiologie intelectuelle; Discurso pro- 
nunciado por el Dr. Foscati el 14 de enero de 1827 en la apertura de una serie de conferencias en la casa de Gall en Paris.

Gall, F. J. (1825). On the origin of the moral qualities and intellectual faculties of man, and the conditions of their manifestation. Boston, Mass: Marsch, Capen and Lyon.

Gallegos, X., Colotla, V. y Jurado, S. (1985). Desarrollo histórico de la psicología en México. Revista de historia de la psicología, (3), 227-239.

Gil, R. (2008). La obra científica del doctor Jesús Díaz de León. Aguascalientes: ICA.

Griessinger, W. (1867). Mental pathology and therapeutics. London: The new Sydenham Society.

Hartley, D. (1834). Observations on man, his fame, his duty, and his expectations. London: Printed for Thomas Tegg and son.

Heineccio, J. (1845/1771). Elementos de filosofía racional, o sea de lógica, traducidos del latín por el Lic. Pedro Ruano. Toluca: Imp. del Instituto Literario.

Hothersall, D. (2005). Historia de la psicología. México: Mc Graw Hill.

Hume, D. (1923). Béchamp or Pasteur, a lost chapter in the history of biology. London: The C. W. Danierl Company.

Jouffroy, T. (1845). Introduction to ethics including a critical survey of moral systems. Boston: James Munroe and Company.

Lafaye, B. (1833). Dissertation sur la philosophie atomistique. Paris: L'imprimerie Royale.

Lares, T. (1849). Elementos de sicología, formados de los diversos artículos sociológicos escritos en francés por C. M. Paffe, Jouffroy, Foscati, Satur, Benjamin Lafaye, Billot, Matter, Loyau D’Amboise, Saigey; y las obras filosóficas de Dougald Stewart. Traducidos al castellano, y ordenados en un cuerpo de doctrina. Para el uso de los alumnos del instituto literario de Zacatecas. Por el Lic. D. Teodiso Lares, director del mismo instituto. Zacatecas: Juan Murphy. López, y De la Peña, X. (2013). Ideas sobre la inmortalidad del alma de Jesús Díaz de Léon Ávila. Aguascalientes: Universidad Autónoma de Aguascalientes.

Magendie, F. (1884). Préis élémentaire de physiologie. Londres: Bruxelles.

Matter, J. (1828). Histoire critique du gnosticisme et de son influence sur les sectes religieuses et philosophiques de six premiers siècles de l'ère chrétienne. Paris: F. G. Levrault.

Melgar Adalid, M. (2011). Teodosio Lares, encargado del despacho. Anuario mexicano de historia del derecho. Vol. 11. Recuperado de: http:// www.juridicas.unam.mx/publica/librev/rev/hisder/cont/7/cnt/cnt7.pdf. 
Menegus, M. (1984). Catálogo de fondo Ezequiel A. Chávez (introducción). México: CESU, Centro de estudio sobre la unAm.

Paffe, C. M. (1832). Considérations Sur La Sensibilité Mise à Sa Place, et présentée comme essentiellement distinct du principe intellectuel. Paris: Chez le Normant.

Pavón Cuéllar, D. (2011). Cinco siglos de posiciones alternativas, críticas y radicales en la psicología mexicana. Athenea digital, 11(2), 193213.

Piaget, J. (1929). The Child's Conception of the World. New York: Harcourt.

Pinel, P. (1800). Traité medico philosophique sur l'aliénation mentale. Paris: Chez. J. Ant. BROSSON.

Saigey, M. (1834). Traite de métrologie ancienne et moderne, suivi d'un précis de chronologie, et des signes numériques. Paris: Libraire classique et élémentaire de L. Hachette.

Saint-Simon, H. (1821). Du système industriel. Paris: Chez Antoine-Augustin Renouard.

Somolinos, G. (1976). Historia de la psiquiatría en México. México: SEP.

Stewart, D. (1854). The collected Works of Dugald Stewart, edited by Sir. William Hamilton. Bart, Edinburgh: Thomas Constable and Co. Hamilton Adams \& Co, London.

Stuart Mill, J. (1872). A system of logic, ratiocinative and inductive, being a connected view of the principles and the methods of scientific investigation. London: Longman's, Green, reader and Dyer.

Titchener, E. (1896). An outline of psychology. New York: The McMillan Company.

Valverde Téllez, E. (1904). Estudio bibliográfico y crítico de las obras de filosofía escritas, traducidas o publicadas en México desde el siglo XVI hasta nuestros días. México: Tipografía de los Sucesores de Francisco Díaz de León. 